\title{
A multicomponent non-pharmacological intervention to prevent delirium for hospitalized people with advanced cancer: a phase Il cluster randomized waitlist controlled trial (the PRESERVE pilot study)
}

\section{Authors}

Annmarie Hosie PhD MPallCarAgeCare RN, ${ }^{1,2,3}$ Jane Phillips PhD RN, ${ }^{3}$ Lawrence Lam PhD Grad Dip Biostats MPH MAppPsych BSc (Hons), ${ }^{3}$ Slavica Kochovska PhD MA BA (Hons), ${ }^{3}$ Beverly Noble, ${ }^{3}$ Meg Brassil, ${ }^{3}$ Susan Kurrle PhD DipGerMed MBBS, ${ }^{4}$ Anne Cumming, ${ }^{5}$ Gideon A Caplan FRACP MBBS MD, ${ }^{6,7}$ Richard Chye FRACP FFPMANZCA FAChPM MBBS, ${ }^{2,7}$ E. Wesley Ely MPH BS MD, ${ }^{8,9}$ Peter G. Lawlor CCFP MBBCh BAO MMed Sc (Pathology), ${ }^{10,11,12}$ Shirley H. Bush FAChPM MRCGP MBBS, ${ }^{10,11,12}$ Jan Maree Davis FRACP FAChPM BMed (Hons), BA(Hons), ${ }^{13}$ Melanie Lovell PhD FRACP FAChPM MBBS, ${ }^{3,14,15}$ Cynthia Parr FAChPM MBBS, ${ }^{14}$ Sally Williams FACRRM FAChPM MBBS PGDipObstMedGyn DipPM, ${ }^{16}$ Katherine Hauser FRACGP FACHPM MBBS (Hons), ${ }^{16}$ Susan McArdle GradDipPallC RN, ${ }^{16}$ Karen Jacquier MPallC Grad Dip Nurs Sc RN, ${ }^{16}$ Carl Phillipson BSW, ${ }^{16}$ Lynne Kuwahata FRAChPM MBBS, ${ }^{17}$ Jackie Kerfoot FRACP MBBCh, ${ }^{17}$ Linda Brown MBus BBus(Mgmt), ${ }^{3}$ Belinda Fazekas BN GrapDipCommHealth, ${ }^{3}$ Seong Leang Cheah PhD MSc BScEd (Hons), ${ }^{3}$ Layla Edwards BAppPH, ${ }^{3}$ Anna Green PhD MDev BSocSci, ${ }^{3}$ Jane Hunt Cert IV Training \& Assessment RN, ${ }^{3}$ Robyn Attwood MHM RN, ${ }^{3}$ Teresa Assen MN BScBEd RN, ${ }^{3}$ Maja Garcia BMedSc(Hons) BIntSt, ${ }^{3}$ Julie Wilcock EN, ${ }^{18}$ Meera Agar, PhD FRACP FAChPM MPallCare MBBS (Hons I) ${ }^{3,18}$

\section{Institutions}

1. The University of Notre Dame Australia, School of Nursing Sydney, Darlinghurst, Australia

2. St Vincent's Health Network Sydney, Darlinghurst, Australia

3. University of Technology Sydney, IMPACCT, Faculty of Health, Ultimo, Australia

4. University of Sydney, Northern Clinical School, Hornsby Ku-ring-gai Health Service, Hornsby, Australia

5. Australian Commission on Safety and Quality in Health Care, Sydney, Australia

6. Prince of Wales Hospital, Geriatric Medicine, Randwick, NSW, Australia

7. University of New South Wales, Randwick, NSW, Australia

8. Critical Illness, Brain Dysfunction, and Survivorship (CIBS) Center, Vanderbilt University, Nashville, TN, USA

9. Tennessee Valley Veteran's Affairs Geriatric Research Education Clinical Center (GRECC), Nashville, TN, USA

10. University of Ottawa, Department of Medicine, Ontario, Canada

11. Ottawa Hospital Research Institute, Ontario, Canada

12. Bruyère Research Institute, Ottawa, Ontario, Canada

13. Calvary Health Care Kogarah Palliative Care, Kogarah, NSW, Australia

14. Greenwich Hospital, HammondCare, Greenwich, NSW, Australia

15. Sydney Medical School, University of Sydney, NSW, Australia

16. The Queen Elizabeth Hospital, Woodville, SA, Australia

17. Camden Hospital, Camden, NSW, Australia

18. Ingham Institute, Liverpool Hospital, Liverpool, NSW, Australia

\section{Corresponding Author}

Dr Annmarie Hosie

Level 3, 235 Jones St. Ultimo NSW 2007

PO Box 123. Broadway NSW 2007 Australia

Phone: +6129514 4404

Email: annmarie.hosie@uts.edu.au

\section{Document details}

Number of tables: 3 Number of figures: 3 Number of references: 38 Word count: 2936 


\section{Background}

Delirium is a common debilitating complication of advanced cancer.

\section{Objective}

To determine if a multicomponent non-pharmacological delirium prevention intervention was feasible for adult patients with advanced cancer, prior to a phase III (efficacy) trial.

\section{Design}

Phase II (feasibility) cluster randomized controlled trial. All sites implemented delirium screening and diagnostic assessment. Strategies within sleep, vision and hearing, hydration, orientation, mobility and family domains were delivered to enrolled patients at intervention sites admission days 1-7. Control sites then implemented the intervention ('waitlist sites').

\section{Setting}

Four Australian palliative care units

\section{Measurements}

The primary outcome was adherence, with an a priori endpoint of at least $60 \%$ patients achieving full adherence. Secondary outcomes were interdisciplinary care delivery, delirium measures and adverse events, analyzed descriptively and inferentially.

\section{Results}

Sixty-five enrolled patients ( 25 control, 20 intervention, 20 waitlist) had $98 \%$ delirium screens and $75 \%$ diagnostic assessments completed. Nurses (67\%), physicians (16\%), allied health (8.4\%), family $(7 \%)$, patients $(1 \%)$ and volunteers $(0.5 \%)$ delivered the intervention. There was full adherence for $5 \%$ patients at intervention sites, partial for $25 \%$. Both full and partial adherence was higher at waitlist sites: $25 \%$ and $45 \%$, respectively. One-third of control site patients (32\%) became delirious within seven days of admission compared to one-fifth (20\%) at both intervention and waitlist sites $(p=0.5)$. Mean (SD) Delirium Rating Scale-Revised-1998 scores were $16.8+12.0$ control sites versus $18.4+8.2(p=0.6)$ intervention and $18.7+7.8(p=0.5)$ waitlist sites. The intervention caused no adverse events.

\section{Conclusion}

The intervention requires modification for optimal adherence in a phase III trial.

\section{Key words}

Cancer, clinical trial, delirium, inpatients, non-pharmacological, palliative care 


\section{Introduction}

Delirium is an acute, distressing and debilitating neurocognitive disorder of physiological etiology for more than one third of patients with advanced cancer in palliative care units. ${ }^{1-4}$ Occurrence is higher again for those who die in this setting, with median (range) period prevalence of $75 \%$ (58\%-88\%). ${ }^{2}$ The high prevalence contributes to widely-held assumptions that delirium is inevitable in people with advanced cancer, especially in the last weeks and days of life. ${ }^{5}$ Yet potential to prevent this devastating complication exists, warranting systematic evaluation in oncology and palliative care contexts..$^{6-8}$

Other clinical settings have decreased delirium incidence and risk in older and seriously ill patients, including intensive care units (ICU) where it was once thought unavoidable. ${ }^{9-14}$ Overall, one-third reduction in delirium incidence has been reported for patients in non-ICU settings though multicomponent interventions targeting physical and cognitive activity, sleep, hydration, vision and hearing. ${ }^{9-11}$ In a study of over 15,000 ICU patients, complete performance of a care bundle to improve pain, awareness, breathing, use of psychoactive medication, delirium detection, mobility and family engagement significantly reduced the likelihood of becoming delirious the following day (adjusted odds ratio $0.60(0.49-0.72) \mathrm{p}<0.0001) .{ }^{14}$

Achieving similar results for people with life-limiting illness in hospital would enable many to maintain mental and physical function and better attend to what and who is most important to them. ${ }^{15}$ We therefore designed a program of research to evaluate whether targeting the fundamental care needs of people with advanced cancer in hospital would improve delirium-related outcomes, including for patients in the last weeks and days of life. In line with the Medical Research Council framework for complex interventions, ${ }^{16}$ we first developed a multicomponent nonpharmacological delirium prevention intervention for patients with advanced cancer from existing evidence $^{4,7,9-11}$ then tested its methods and impact in a phase II (feasibility) trial, prior to embarking on a phase III (efficacy) trial of the intervention.

Results of the phase II trial are reported here according to Consolidated Standards of Reporting Trials guidelines. ${ }^{17}$ The trial was registered with the Australian and New Zealand Clinical Trials Registry ${ }^{18}$ and a protocol detailing its justification and methods was published in January 2019. ${ }^{19}$

\section{Methods}

Design

A phase II cluster randomised controlled trial (RCT). We chose the cluster RCT design because the intervention suited site-level implementation and it reduced risk of contamination. As similar interventions have been effective in other patient populations and settings, site investigators 
requested that control sites be given opportunity to later trial the intervention. We therefore integrated a 'waitlist' option for control sites into the study design, whereby sites randomized to control were given details of the intervention and option to deliver it after initial data collection at intervention sites. ${ }^{20}$ Addition of the waitlist option furthermore provided opportunity to refine the intervention and/or study processes if initial data from intervention sites indicated need to do so.

We also embedded a qualitative sub-study to obtain patient, family caregiver, staff and volunteer perspectives about feasibility and acceptability of the intervention and study measures, analysed according to the Theoretical Domains Framework for health-related behaviours. ${ }^{21}$ Methods and findings of the sub-study will be reported in full elsewhere.

The overall study design is presented in figure 1.

\section{Setting and participants}

Participating sites were four specialist palliative care inpatient units within hospitals in metropolitan Australia. Investigators MA and AH recruited sites in 2017. Patients eligible for enrolment were adults (i.e. aged 18 years or over) with advanced (stage 4 ) cancer. ${ }^{22}$

\section{Randomisation}

The trial statistician LL allocated sites to intervention or control plus waitlist intervention conditions in October 2017 at UTS using a permuted block randomization method. We notified sites of allocation after local governance approvals.

\section{Ethical procedures}

Hospital and university Human Research Ethics Committees approved the trial (HREC/17/LPOOL/224 and ETH17-1697). One person per site with requisite authority gave cluster-level consent to participate. Patients and family were informed about the trial via posters and verbal communication, including that they could opt out.

\section{Intervention and control conditions}

All sites instituted delirium screening and diagnostic assessment of enrolled patients for the first seven days of admission. Nurses completed the Nursing Delirium Screening Scale (Nu-DESC) ${ }^{23}$ each eight-hour shift. Within 24 hours of a positive Nu-DESC (score $\geq 2$ ) for patients not already diagnosed as delirious, physicians applied Diagnostic and Statistical Manual of Mental Disorders, Fifth edition (DSM-5) diagnostic criteria for delirium ${ }^{1}$ and the Delirium Rating Scale-Revised-1998 (DRS-R-98). ${ }^{24}$ Except for one site that mandated the Nu-DESC for the first three days of admission, structured delirium screening and diagnostic assessment were new processes and therefore additional to usual care. 
The intervention had six domains (eating and drinking, sleep, exercise, reorientation, vision and hearing, and family partnership) containing 36 strategies overall (4-12 per domain) (supplementary file 1). We asked team members to enlist family and volunteers and tailor the intervention to patients' needs and wishes. Team members documented delivered strategies on a checklist designed for data collection, plus their designation and reason for non-delivery: 'not required', 'patient choice', 'not clinically appropriate', 'not possible with current resources', and 'other'. ${ }^{19}$ A two-month site engagement and training period, guided by customized information manuals (available on request), preceded control and intervention conditions. Sites formed working groups of interested team members to plan implementation in line with their resources and systems. University-based researchers attended working group meetings to ensure intervention fidelity, trial integrity and timely progress $[\mathrm{AH}, \mathrm{RA}, \mathrm{JH}, \mathrm{JW}]$. Sites shared meeting records whenever researchers could not attend in person.

We designed and conducted training via four discrete 30-40 minute sessions (i.e. Introduction to the study; Delirium screening using the NuDESC; Delirium assessment and diagnosis; Multicomponent delirium prevention intervention) using Biggs' educational mode ${ }^{25}[\mathrm{AH}, \mathrm{RA}, \mathrm{JH}, \mathrm{JW}$, site investigators, clinical educators, nursing managers], delivered multiple times for broadest reach. Learning outcomes were clinicians' understanding of the study processes relevant to their role and achievement of target adherence to study measures. Control sites received information about delirium prevention strategies when they transitioned to the intervention phase, along with a summary of learnings from intervention sites about optimizing trial processes. A key message was that the checklist was not the intervention per se, but essential to measuring the primary outcome of adherence.

\section{Data collection}

Site research nurses recorded delirium measures, intervention delivery and adverse events during enrolled patients' first seven days of admission on Case Report Forms and Excel spreadsheets and uploaded data into REDCap. ${ }^{26}$ Project staff obtained site-level patient demographics and functional status for all patients admitted during study periods from Palliative Care Outcomes Collaboration datasets. ${ }^{27}$

The schedule of study measures is presented in supplementary file 2 .

\section{Blinding}

The cluster design and nature of the intervention precluded blinding of site teams and data collectors. To minimize ascertainment bias, sites teams provided patients and family caregivers with 
information about clinical aspects of the trial but not design or site allocation. We used standardized data collection tools and training to limit measurement bias.

\section{Outcomes}

The primary outcome was intervention adherence at intervention and waitlisted sites, according to checklist documentation of each strategy at least once daily for the first seven days of admission. $A$ priori, $60 \%$ or more patients having full adherence (delivery of all required strategies within at least four domains for at least five days) was considered minimum evidence that the intervention was feasible without major modification. Partial adherence to the intervention was defined as at least one strategy in at least four domains daily for at least five days.

Secondary outcomes were:

1. Delivery of care: i) Sample feasibility (percentage of patients with data collected, reasons for non-inclusion, time to achieve sample); ii) Coverage (proportion eligible patients receiving intervention, reasons for non-delivery, weekend coverage); iii) Compliance with study measures; iv) Methods, areas and levels interdisciplinary delivery; and v) Sustainability (adherence to delirium measures and prevention strategies at sites six months after intervention);

2. Delirium-related: i) Proportion of patients with a positive Nu-DESC at least once each 24hours; ii) Delirium incidence (first meeting DSM-5 delirium diagnostic criteria within 24hours of a positive Nu-DESC); and iii) Delirium severity (DRS-R-98 at first onset);

3. Adverse events: Falls, deaths, other, according to National Cancer Institute Common Terminology Criteria for Adverse Events ${ }^{28}$ and complaints; reported as numbers, rates and descriptively, overall and intervention-related.

\section{Sample size}

To obtain a sufficient sample size for a pilot study of at least $9 \%$ of the sample of the planned phase III trial, we enrolled four sites and 40 patients (10 per site), then 20 patients at waitlisted sites (10 per site). ${ }^{29}$

\section{Statistical analysis}

Adherence to the delirium prevention strategies and by whom was computed using conditional counting techniques. Secondary outcomes were summarized using frequency and percentage for categorical variables, and mean and standard deviation or median and range for continuous variables. Independent sample t-tests, Chi-square tests and Fisher's Exact Test were used to measure differences between patients' demographic and delirium status across control, intervention and 
waitlist sites. Data were managed using Microsoft Excel 2016 and Statistical Package for Social Sciences version 25 .

\section{Results}

During enrolment periods, the four sites admitted 90 patients and screened all for eligibility (figure 2). Seventy-two patients were eligible according to the protocol, with 68 considered eligible by site investigators. Four eligible patients were not enrolled due to: 'distress on admission', 'patient admitted for terminal care', 'patient unable to communicate well', with no reason given for the fourth. Non-enrolment of three patients considered eligible by site investigators was because two patients requested withdrawal and one 'other'.

We collected and analyzed data for 65 patients (90\% of protocol-eligible patients, $96 \%$ according to site investigators). Of these, 38 patients (58\%) had seven days of data collected. Twenty patients died within seven days (31\%), four were discharged (6\%), one control site patient was considered not well enough to continue, and two patients had data collection ceased before seven days for other, non-specified reasons.

Patient enrolment took an average of 20 days per site (range 8-36) and the overall sample was achieved in five months (May-September 2018).

\section{Site-level patient characteristics}

During study periods, just over half (56\%) of all admitted patients were male. Most were aged 70 years or over (59\%), with a primary diagnosis of cancer (90\%), born in Australia (56\%) and had English their primary language (92\%). Few were Aboriginal or Torres Strait Islander ( $n=2,1 \%)$. There were no significant differences in patients' baseline characteristics at control sites compared to intervention or waitlist sites (table 1).

\section{Adherence to the intervention}

Overall, 40 patients received the intervention for an average (SD) of $5.8 \pm 1.8$ days. Of these, fourteen died (35\%) and two (5\%) were discharged within seven days of admission.

There was full adherence for $5 \%$ patients at intervention sites, $25 \%$ at waitlist sites. Percentages of patients having partial adherence was $25 \%$ at intervention sites, $45 \%$ at waitlist sites (figure 3 ).

Per domain, highest adherence was to exercise (59\%), then eating and drinking (54\%), reorientation (52\%), and sleep, vision and hearing and enabling family (each 41\%) (table 2). There was higher delivery of every strategy at waitlist sites. Overall, nurses delivered most strategies (67\%), followed by medical staff $(16 \%)$, allied health $(8.4 \%)$, family caregivers $(7 \%)$, volunteers $(0.5 \%)$, with patients themselves recording $1.1 \%$. There was minimal documentation of reasons for non-delivery: 'not 
required' (1.4\%), 'not appropriate' (1\%), 'patient choice' (0.7\%), 'not possible' $(0.4 \%)$, and 'other' $(n=1)$.

\section{Delirium measures}

Nurses completed the Nu-DESC at least once per day for $373 / 379$ patient days (98\%). Physicians applied the DSM-5 diagnostic criteria and DRS-R-98 within 24-hours of a first positive Nu-DESC for 24/31 occasions (77.4\%). There was no significant difference between weekday and weekend completion of delirium measures.

Eight patients developed delirium in the first seven days of admission at control sites (32\%) compared with four patients each at intervention (20\%) and waitlist sites $(20 \%)(p=0.5)$. Mean (SD) delirium severity (DRS-R-98) was $16.8 \pm 12.0$ for patients at control sites compared with $18.4 \pm 8.2$ at intervention sites $(p=0.6)$ and $18.7 \pm 7.8$ at waitlist sites $(p=0.5)$ (table 3 ).

\section{Adverse events}

Forty-five patients (69\%) experienced a total of 162 adverse events: 15 (60\%) control, 14 (70\%) intervention and 16 (80\%) waitlist sites (supplementary table 3). Site investigators attributed no adverse events to the intervention. Two (8\%) control site patients fell, compared with one each at intervention and waitlist sites (5\%). Six (24\%) patients died within seven days of admission at control sites compared to seven (35\%) at both intervention and waitlist sites.

\section{Sustainability (at six months)}

Of 20 consecutively admitted patients (five per site), two patients (10\%) died within five days, leaving 97 patient days for analysis. In these, there were five instances of delirium screening using the NuDESC and one using the Confusion Assessment Method (6\%). ${ }^{30}$ There was no DRS-R-98 or DSM-5 diagnostic criteria for delirium documented. Adherence to the delirium prevention strategies was not full for any patient and partial for six (30\%). Almost every strategy was less frequently documented than during the intervention (table 2).

\section{Discussion}

Despite site engagement and training and higher intervention adherence at waitlist sites, the trial did not achieve its primary feasibility outcome. In contrast, secondary outcomes demonstrated feasibility of the sample and delirium measures, no adverse effects, and a non-statistically significant decrease in the proportion of patients with delirium in the first week of admission when sites delivered the intervention. Adherence was higher at waitlist sites, likely due to the more staged implementation (i.e. delirium measures then prevention strategies), in conjunction with giving waitlist site teams more explicit information about importance of documenting the intervention. 
Post-intervention, measurement of usual care demonstrated higher adherence to strategies occurred within-trial. Taken together, results support the ongoing investigation of delirium prevention in patients with advanced cancer though non-pharmacological means. However, as higher delivery of multicomponent non-pharmacological delirium prevention interventions is associated with greater effectiveness, modifications to optimize adherence in a phase III trial will be required. ${ }^{14,31}$

Next steps in this staged research program are to integrate qualitative findings with trial results to inform modification of the intervention. Strategies to be retained will be those with highest overall evidence, delivered with higher adherence or under-delivered due to resolvable barriers in this trial, and considered essential by those involved.

An intervention with fewer strategies will also facilitate more precise dose measurement. In this feasibility trial, it was sufficient to measure intervention frequency (24 hours) and duration (one week), whereas determining dose/efficacy ratios will also require measuring the amount patients receive each time..$^{32}$ Full dose measurement will require a priori consideration of equivalent strengths of intervention parts and whether to measure these at the domain or strategy level. Other factors important to consider for dose potency are who delivers the strategy and how, because fundamental care strategies are delivered through highly interpersonal interactions. Such interactions serve not only visible objectives (e.g. to help the person drink a glass of water) but also to communicate caring, compassion and respect for an ill person. How fundamental care is delivered is instrumental to maintenance of psychological well-being during advanced illness and dying in hospital and therefore worthy of closer attention in future delirium research in palliative care contexts. ${ }^{33,34}$

We explicitly sought to include patients near the end of life in this trial and so it was not surprising that almost one-third of enrolled patients died within seven days of admission. Endeavouring to develop and trial non-pharmacological delirium interventions in the last weeks of life is justifiable, because patients with terminal illness value maintaining cognition and connection with others ${ }^{35,36}$ and both delirium and customary pharmacological treatments disrupt this potential. ${ }^{8,37}$ However, it is not possible to diagnose delirium in an unresponsive person. ${ }^{1}$ Therefore, collateral measurement of psychomotor activity and awareness would inform a primary trial outcome of days without delirium or coma, as successfully evaluated in studies in intensive care. ${ }^{12,14}$ This would be a clinically meaningful palliative care outcome, as it would help determine the extent to which nonpharmacological delirium interventions enable patients to remain more alert and able to attend to important undertakings with those they love in the last days of life. ${ }^{38}$ 


\section{Limitations and strengths}

Limitations include lack of blinding, inherently difficult in multicomponent non-pharmacological interventions and a source of bias in this field of delirium research. ${ }^{7}$ The small sample was appropriate for a phase II trial but precluded definitive determination of intervention effectiveness in reducing delirium incidence or severity. While patients who became delirious continued to receive the intervention, we did not examine delirium treatment or resolution. Strengths include the cluster RCT design, which promoted enrolment of almost all eligible patients and reduced the risk of selection bias. The waitlisted arm facilitated multisite recruitment and engagement. Systematic adverse event measurement ensured close monitoring of enrolled patients for potential harms and was an advancement upon previous similar studies. ${ }^{7}$

\section{Generalisability}

The study informs future research in delirium prevention in patients with advanced cancer in palliative care units. The study population was representative of Australian palliative care inpatients and sites typified the varyingly resourced units across metropolitan Australia. Intervention strategies addressed fundamental human needs, and prior implementation in many settings underscores their universal relevance for hospitalized patients. ${ }^{15-17}$ However, results may not be generalized to other geographical regions, health care systems or patients with other advanced illnesses.

\section{Conclusion}

A multicomponent intervention to optimize eating and drinking, sleep, exercise, reorientation, vision and hearing and family partnership to prevent delirium in patients with advanced cancer was piloted in four palliative care units. Outcomes indicate a phase III trial will be feasible in this patient population and setting if the intervention is modified. Next steps are to integrate qualitative findings with trial results to inform modification of the intervention, and determine dose and outcome measures most fitting for patients with advanced cancer in palliative care units, including those in the last weeks and days of life. 


\section{Acknowledgements}

The authors gratefully acknowledge the contributions of patients, family caregivers, clinicians and volunteers at the four participating sites and the Palliative Care Outcomes Collaboration team who provided site level data.

The trial was funded by an Australian National Breast Cancer Foundation (NBCF) 2017 Pilot Study Grant (Grant code PS-17-030), contact details Level 9, 10 Barrack Street, Sydney, NSW, 2000, Australia; T: +61 280984800 E: info@ nbcf.org.au, W: https://nbcf.org.au/. The NBCF had no role in the study design, data collection, management, analysis, interpretation or publication of results. The trial sponsor was the Palliative Care Clinical Trials Collaborative (PaCCSC) (Level 3, 235 Jones St, Ultimo, NSW, 2007, Australia; T. +61 (2) 95144862 (Sydney) /+61 (8) 74219726 (Adelaide), E: paccsc@uts.edu.au, W: uts.edu.au/paccsc). PaCCSC supports optimal trial governance through standard operating procedures for electronic data handling, completion of case report forms, monitoring, dissemination, archiving of research materials, and record destruction, and trial infrastructure through Trials Management and Scientific Committees.

\section{Author Disclosure Statement}

Drs. Hosie, Phillips, Lam, Kochovska, Cheah, Green and Agar and Ms Brown, Fazekas Edwards, Hunt, Attwood, Assen, Garcia and Wilcock report a grant from the National Breast Cancer Foundation during the conduct of the study. Dr Hosie also reports personal fees from Medtronic, outside the submitted work. Dr. Caplan reports grants from Bionomics Pty Ltd, outside the submitted work. Dr. Ely reports personal fees from Masimo, grants from $\mathrm{VA} / \mathrm{NIH}$, personal fees from Pfizer/Orion and grants from Koheler, outside the submitted work. All remaining authors have no disclosures to report.

\section{Authors' contributions}

$\mathrm{AH}, \mathrm{MA}, \mathrm{JP}$ co-led the trial and $\mathrm{AH}$ is corresponding author. MA, JP, AH developed the intervention in consultation with an expert working group. ${ }^{19} \mathrm{MB}, \mathrm{BN}$ provided consumer insights into trial rationale and the intervention. $\mathrm{AC}$ advised on alignment of the intervention and delirium measures with the Australian Commission on Safety and Quality in Health Care's Delirium Clinical Care Standard. LL devised the statistical analysis and randomization process. JMD and $\mathrm{ML}$ provided insights into the waitlist design. AH, MA, JMD, ML, CP, SW, KH, SMcA, KJ, CP, LK, JK, JH, RA, TA, MG, JW co-designed processes for site engagement, training and intervention implementation. SK, GC, RC, BL, EWE, PL, SB contributed clinical and research expertise into study design, process, measures and/or analysis. $L B, B F, A G, S L C$, LE contributed to processes for data collection, adverse event reporting entry, storage and/or analysis. All authors have approved manuscript submission. 


\section{References}

1. American Psychiatric Association: Diagnostic and Statistical Manual of Mental Disorders, Fifth Edition (DSM-5). Arlington, VA: American Psychiatric Publisher; 2013.

2. Watt $\mathrm{CL}$, Momoli F, Ansari MT, et al.: The incidence and prevalence of delirium across palliative care settings: A systematic review. Palliative Medicine. 2019;33(8):865-877.

3. O'Malley $\mathrm{G}$, Leonard M, Meagher D, et al.: The delirium experience: a review. Journal of Psychosomatic Research. 2008;65(3):223-228.

4. Finucane AM, Lugton J, Kennedy C, et al.: The experiences of caregivers of patients with delirium, and their role in its management in palliative care settings: an integrative literature review. Psycho-Oncology. 2017;26(3):291-300.

5. Hosie A, Agar M, Phillips J: Re-thinking our approach to care of the dying person with delirium: time for a new care paradigm. International Journal of Palliative Nursing. 2016;22(10):472-473.

6. Lawlor PG, Rutkowski NA, MacDonald AR, et al.: A Scoping Review to Map Empirical Evidence Regarding Key Domains and Questions in the Clinical Pathway of Delirium in Palliative Care. Journal of Pain and Symptom Management. 2019;57(3):661-681.e612.

7. Hosie A, Siddiqi N, Featherstone I, et al.: Inclusion, characteristics and outcomes of people requiring palliative care in studies of non-pharmacological interventions for delirium: A systematic review. Palliative Medicine. 2019;33(8):878-899.

8. Agar M, Lawlor PG, Quinn S, et al.: Efficacy of oral risperidone, haloperidol, or placebo for symptoms of delirium among patients in palliative care: A randomized clinical trial. JAMA Internal Medicine. 2017;177(1):34-42.

9. Siddiqi N, Harrison JK, Clegg A, et al.: Interventions for preventing delirium in hospitalised non-ICU patients. Cochrane Database of Systematic Reviews. 2016 (Issue 3. Art. No.: CD005563. ).

10. Hshieh TT, Yue J, Oh E, et al.: Effectiveness of multicomponent nonpharmacological delirium interventions: a meta-analysis. JAMA Internal Medicine. 2015;175(4):512-520.

11. Abraha I, Trotta F, Rimland JM, et al.: Efficacy of Non-Pharmacological Interventions to Prevent and Treat Delirium in Older Patients: A Systematic Overview. The SENATOR project ONTOP Series. PLOS ONE. 2015;10(6):e0123090.

12. Girard TD, Exline MC, Carson SS, et al.: Haloperidol and Ziprasidone for Treatment of Delirium in Critical IIIness. New England Journal of Medicine. 2018;379:2506-2516.

13. Ely EW: The ABCDEF Bundle: Science and Philosophy of How ICU Liberation Serves Patients and Families. Critical Care Medicine. 2017;45(2):321-330.

14. Pun BT, Balas MC, Barnes-Daly MA, et al.: Caring for Critically III Patients with the ABCDEF Bundle: Results of the ICU Liberation Collaborative in Over 15,000 Adults. Critical Care Medicine. 2018;47(1):3-14.

15. McCaffrey N, Skuza P, Breaden K, et al.: Preliminary Development and Validation of a New End-of-Life Patient-Reported Outcome Measure Assessing the Ability of Patients to Finalise Their Affairs at the End of Life. PLOS ONE. 2014;9(4):e94316.

16. Craig P, Dieppe $P$, Macintyre $S$, et al.: Developing and evaluating complex interventions: the new Medical Research Council guidance. BMJ. 2008;337:a1655.

17. Campbell MK, Piaggio G, Elbourne DR, et al.: Consort 2010 statement: extension to cluster randomised trials. BMJ: British Medical Journal. 2012;345:e5661.

18. Agar M, Hosie A: Phase 2 cluster randomized controlled trial of a multi-component nonpharmacological intervention to prevent delirium for hospitalized people with advanced cancer: The PRESERVE pilot study (ACTRN12617001070325p). 2017; https://www.anzctr.org.au/Trial/Registration/TrialReview.aspx?id=373168. Accessed November 18, 2019. 
19. Hosie A, Phillips J, Lam L, et al.: Multicomponent non-pharmacological intervention to prevent delirium for hospitalised people with advanced cancer: study protocol for a phase II cluster randomised controlled trial. BMJ Open. 2019;9(1):e026177.

20. Higginson IJ, Booth S: The randomized fast-track trial in palliative care: Role, utility and ethics in the evaluation of interventions in palliative care? Palliative Medicine.

2011;25(8):741-747.

21. Atkins L, Francis J, Islam R, et al.: A guide to using the Theoretical Domains Framework of behaviour change to investigate implementation problems. Implementation Science. 2017;12(1):77.

22. Cancer Institute NSW: What are the different stages of cancer? 2019; https://www.cancer.nsw.gov.au/learn-about-cancer/what-are-the-different-stages-ofcancer. Accessed November 18, 2019.

23. Gaudreau J-D, Gagnon P, Harel F, et al.: Fast, Systematic, and Continuous Delirium Assessment in Hospitalized Patients: The Nursing Delirium Screening Scale. Journal of Pain and Symptom Management. 2005;29(4):368-375.

24. Trzepacz PT, Mittal D, Torres R, et al.: Validation of the Delirium Rating Scale-revised-98: comparison with the delirium rating scale and the cognitive test for delirium. The Journal of Neuropsychiatry and Clinical Neurosciences. 2001;13(2):229-242.

25. Walsh A: An exploration of Biggs' constructive alignment in the context of work-based learning. Assessment \& Evaluation in Higher Education. 2007;32(1):79-87.

26. Harris PA, Taylor R, Thielke R, et al.: Research electronic data capture (REDCap)--a metadatadriven methodology and workflow process for providing translational research informatics support. Journal of Biomedical Informatics. 2009;42(2):377-381.

27. Palliative Care Outcomes Collaboration. 2019; https://ahsri.uow.edu.au/pcoc/index.html. Accessed November 18, 2019.

28. National Institutes of Health NCI: 2010. NIH Publication No. 09-5410 [Common Terminology Criteria for Adverse Events (CTCAE) v4.03;

http://www.hrc.govt.nz/sites/default/files/CTCAE\%20manual\%20-\%20DMCC.pdf. Accessed November 18, 2009.

29. Cocks K, Torgerson DJ: Sample size calculations for pilot randomized trials: a confidence interval approach. Journal of Clinical Epidemiology. 2013;66(2):197-201.

30. Inouye SK, van Dyck CH, Alessi CA, et al.: Clarifying Confusion: The Confusion Assessment Method: A New Method for Detection of Delirium. Annals of Internal Medicine. 1990;113(12):941-948.

31. Inouye SK, Bogardus ST, Jr., Williams CS, et al.: The role of adherence on the effectiveness of nonpharmacologic interventions: evidence from the delirium prevention trial. Archives of Internal Medicine. 2003;163(8):958-964.

32. Reed D, Titler MG, Dochterman JM, et al.: Measuring the Dose of Nursing Intervention. International Journal of Nursing Terminologies and Classifications. 2007;18(4):121-130.

33. Collier A, De Bellis A, Hosie A, et al.: Fundamental care for people with cognitive impairment in the hospital setting: A study combining positive organisational scholarship and videoreflexive ethnography. Journal of Clinical Nursing. 2019;0(0).

34. Spichiger E: Living with terminal illness: patient and family experiences of hospital end-of-life care. International Journal of Palliative Nursing. 2008;14(5):220-228.

35. Steinhauser KE, Christakis NA, Clipp EC, et al.: Factors Considered Important at the End of Life by Patients, Family, Physicians, and Other Care Providers. JAMA. 2000;284(19):24762482.

36. Delgado-Guay MO, Rodriguez-Nunez A, De la Cruz VJ, et al.: Advanced cancer patients' priorities regarding wishes at the end of life: A randomized controlled study. Journal of Clinical Oncology. 2015;33(29_suppl):44-44. 
37. Hui D, Frisbee-Hume S, Wilson A, et al.: Effect of Lorazepam With Haloperidol vs Haloperidol Alone on Agitated Delirium in Patients With Advanced Cancer Receiving Palliative Care: A Randomized Clinical Trial. JAMA. 2017;318(11):1047-1056.

38. Pandharipande PP, Ely EW: Humanizing the Treatment of Hyperactive Delirium in the Last Days of Life. JAMA. 2017;318(11):1014-1015. 
Table 1: Site-level patient characteristics on admission

\begin{tabular}{|c|c|c|c|}
\hline & $\begin{array}{l}\text { Control sites } \\
(n=2)\end{array}$ & $\begin{array}{l}\text { Intervention sites } \\
(n=2)\end{array}$ & $\begin{array}{l}\text { Waitlisted sites } \\
(n=2)\end{array}$ \\
\hline Patient $\mathrm{n}(\%)$ & $\mathrm{n}=37 * *$ & $\mathrm{n}=56 * *$ & $\mathrm{n}=50 * *$ \\
\hline Age in years, mean (SD) & $70.5(15.5)$ & $76.0(11.2)$ & $68.1(12.5) *$ \\
\hline Aged $>70$ & $19(51)$ & $41(73)$ & $24(48) *$ \\
\hline Male sex & $24(65)$ & $23(41)$ & $33(66) *$ \\
\hline \multicolumn{4}{|l|}{ ATSI status } \\
\hline Not ATSI & $34(92)$ & $56(100)$ & $48(96)$ \\
\hline ATSI & $1(3)$ & 0 & $1(2)$ \\
\hline Not documented & $2(5)$ & 0 & $1(2)$ \\
\hline \multicolumn{4}{|l|}{ Country of Birth } \\
\hline Australia & $23(62)$ & $28(50)$ & $29(58)$ \\
\hline Elsewhere & $11(30)$ & $27(48)$ & $21(42)$ \\
\hline Not documented & $3(8)$ & $1(2)$ & $y$ \\
\hline \multicolumn{4}{|l|}{ Primary language } \\
\hline English & $33(89)$ & $50(89)$ & $49(98)$ \\
\hline Other than English & $2(5.5)$ & $6(11)$ & $1(2)$ \\
\hline Not documented & $2(5.5)$ & 0 & 0 \\
\hline \multicolumn{4}{|l|}{ Primary diagnosis } \\
\hline Malignant & $36(97)$ & $48(86)$ & $45(90)$ \\
\hline Non-malignant & $1(3)$ & $8(14)$ & $5(10)$ \\
\hline \multicolumn{4}{|l|}{ AKPS } \\
\hline 70 & $1(3)$ & $1(2)$ & $1(2)$ \\
\hline 60 & $6(16)$ & $7(12)$ & $1(2)$ \\
\hline 50 & $5(14)$ & $13(23)$ & $10(20)$ \\
\hline 40 & $3(8)$ & $15(27)$ & $15(30)$ \\
\hline 30 & $4(11)$ & $11(20)$ & $5(10)$ \\
\hline 20 & $16(43)$ & $8(14)$ & $16(32)$ \\
\hline \multirow{2}{*}{$\begin{array}{l}10 \\
\text { Total RUG-ADL }\end{array}$} & $2(5)$ & $1(2)$ & $2(4)$ \\
\hline & \multicolumn{3}{|c|}{ Total RUG-ADL } \\
\hline 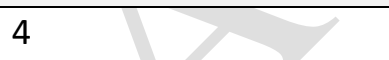 & 7 (19) & $9(16)$ & $3(6)$ \\
\hline $4-18$ & $16(43)$ & $31(55)$ & $37(74)$ \\
\hline 18 & $14(38)$ & $16(29)$ & $10(20)$ \\
\hline
\end{tabular}

AKPS Australian-modified Karnofsky Performance Scale ATSI: Aboriginal or Torres Strait Islander RUG-ADL Resource Utilisation Group Activities Daily Living

* With Bonferroni adjustment for familywise corrections ( $p=0.05 / 3=0.0167)$, independent sample t-test revealed statistical significant difference for mean ages $(p=0.001)$, and Chi-square tests revealed statistical significant differences for Age>70 (0.008) and genders (0.01) between the intervention and waitlisted sites.

* * Site-level data includes ALL patients admitted during study periods 
Table 2: Adherence and interdisciplinary delivery of the intervention

\begin{tabular}{|c|c|c|c|c|c|c|}
\hline Domain (\%) & Strategy (\%) & $\mathrm{I}=20$ & $\begin{array}{l}\mathbf{W} \\
\mathrm{n}=20\end{array}$ & $\begin{array}{l}\text { Overall } \\
n=40\end{array}$ & Delivered by (\%) & $\begin{array}{l}\text { Post } \\
\text { trial } \\
n=20\end{array}$ \\
\hline \multirow[t]{3}{*}{ Exercise (59) } & $\begin{array}{l}\text { Minimize physical restraints e.g. bed rails, } \\
\text { lock-in chair tables }\end{array}$ & 55 & 70 & 63 & \multirow[t]{3}{*}{$\begin{array}{l}\text { Nursing (63.2), allied health }(P, D)^{*}(18.6) \text {, medical (14.9), family (2.6), } \\
\text { patient (0.7) }\end{array}$} & 45 \\
\hline & $\begin{array}{l}\text { Minimize tethers e.g. IV, IDC, drain, oxygen } \\
\text { tubing }\end{array}$ & 55 & 65 & 60 & & 49 \\
\hline & Encourage and assist physical activity & 50 & 60 & 55 & & 77 \\
\hline \multirow{5}{*}{$\begin{array}{l}\text { Eating \& drinking } \\
\text { (54) }\end{array}$} & Encourage oral fluids & 55 & 70 & 63 & \multirow{5}{*}{$\begin{array}{l}\text { Nursing (69.2), family (13.4), medical (10.1), allied health (D, OT, PC, SP)* } \\
\text { (5.5), patient (1.6), volunteer (0.3) }\end{array}$} & 41 \\
\hline & Drinking aids & 40 & 65 & 53 & & 9 \\
\hline & $\begin{array}{l}\text { Respond to reversible causes of poor oral } \\
\text { intake }\end{array}$ & 35 & 70 & 53 & & 21 \\
\hline & Volunteer assist with set up & 40 & 65 & 53 & & 7 \\
\hline & Physical assistance with meals and drinks & 40 & 60 & 50 & & 22 \\
\hline \multirow[t]{12}{*}{ Reorientation (52) } & Greet patient by name & 55 & 70 & 63 & \multirow{12}{*}{$\begin{array}{l}\text { Nursing (66.4), medical (20.6), allied health (D, PC, P, SW, OT)* (8.6), family } \\
\text { (3.4), volunteer (0.8), patient }(0.2)\end{array}$} & 73 \\
\hline & Introduce self & 55 & 70 & 63 & & 74 \\
\hline & Refer to person, time and place & 55 & 70 & 63 & & 75 \\
\hline & Remains in allocated room & 55 & 70 & 63 & & 53 \\
\hline & Discuss current events & 55 & 70 & 63 & & 55 \\
\hline & Encourage patient to reminisce and talk & 55 & 70 & 63 & & 42 \\
\hline & Clock or watch in room & 50 & 70 & 60 & & 99 \\
\hline & Daily update of in-room whiteboards & 45 & 70 & 58 & & 0 \\
\hline & Access to cognitively stimulating activities & 35 & 55 & 45 & & 22 \\
\hline & Translation aids & 15 & 50 & 33 & & 5 \\
\hline & Interpreter & 15 & 45 & 30 & & 1 \\
\hline & Cognitively stimulating activities in care plan & 10 & 40 & 25 & & 0 \\
\hline \multirow[t]{6}{*}{ Sleep (41) } & Lights off or minimized at night & 55 & 70 & 63 & \multirow{6}{*}{$\begin{array}{l}\text { Nursing }(75.6) \text {, medical }(10.9) \text {, allied health (D, PC, OT, P)* (7), family (4.7), } \\
\text { patient (1.6), volunteer (0.2) }\end{array}$} & 41 \\
\hline & Curtains/blinds open in day & 55 & 70 & 63 & & 49 \\
\hline & Uninterrupted sleep during night & 35 & 55 & 45 & & 42 \\
\hline & Time spent outside during day & 30 & 45 & 38 & & 31 \\
\hline & Avoid caffeine after $4 \mathrm{pm}$ & 30 & 45 & 38 & & 11 \\
\hline & Eye shades & 5 & 40 & 23 & & 0 \\
\hline
\end{tabular}




\begin{tabular}{|c|c|c|c|c|c|c|}
\hline & Ear plugs & 5 & 40 & 23 & & 0 \\
\hline \multirow{5}{*}{$\begin{array}{l}\text { Vision \& hearing } \\
\text { (41) }\end{array}$} & Use visual aids & 35 & 65 & 50 & \multirow{5}{*}{$\begin{array}{l}\text { Nursing (64.6), medical (16.5), family (10.7), allied health (D, P, PC, OT, SW, } \\
\text { SP)* (5.1), patient (2.4), volunteer (0.7) }\end{array}$} & 24 \\
\hline & Assess hearing & 30 & 65 & 48 & & 4 \\
\hline & Assess vision & 20 & 60 & 40 & & 4 \\
\hline & Use hearing aids & 25 & 50 & 38 & & 0 \\
\hline & Ear wax clearing & 15 & 40 & 28 & & 0 \\
\hline \multirow{4}{*}{$\begin{array}{l}\text { Enabling Family } \\
\text { (41) }\end{array}$} & Ask about patient's baseline cognition & 30 & 65 & 48 & \multirow[t]{4}{*}{ Nursing (46.6), medical (43.5), allied health (5.3), family (4.6) } & 15 \\
\hline & Invite participation in prevention strategies & 25 & 60 & 43 & & 1 \\
\hline & $\begin{array}{l}\text { Verbally inform about delirium risk and } \\
\text { prevention strategies }\end{array}$ & 20 & 55 & 38 & & 1 \\
\hline & Give delirium brochure & 20 & 55 & 38 & & 0 \\
\hline
\end{tabular}

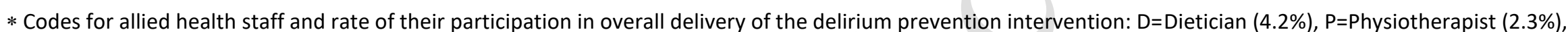
$\mathrm{PC}=$ Pastoral care $(1.1 \%), \mathrm{OT}=$ Occupational therapist $(0.4 \%), \mathrm{SW}=$ Social worker $(0.3 \%), \mathrm{SP}=$ Speech pathologist $(0.1 \%) \mathrm{I}:$ Intervention sites, WI: Waitlist sites

NB all figures are percentages of patients receiving intervention elements 
The PRESERVE pilot study

Table 3: Delirium incidence and severity

\begin{tabular}{|l|l|l|l|}
\hline Delirium measure $\mathrm{n}(\%)$ & $\begin{array}{l}\text { Control sites } \\
(\mathrm{n}=25)\end{array}$ & $\begin{array}{l}\text { Intervention sites } \\
(\mathrm{n}=20)\end{array}$ & $\begin{array}{l}\text { Waitlisted sites } \\
(\mathrm{n}=20)\end{array}$ \\
\hline Nu-DESC $\geq 2$ & $13(52)$ & $6(30)$ & $12(60)$ \\
\hline Delirium incidence (DSM-5) & $8(32)$ & $4(20)$ & $4(20)$ \\
\hline $\begin{array}{l}\text { Delirium severity (DRS-R-98) } \\
\text { Mean (Range; SD) }\end{array}$ & $16.8(2-36 ; 12.0)$ & $18.4(5-24 ; 8.2)$ & $18.7(10-31,7.8)$ \\
\hline
\end{tabular}

Fisher's Exact Test and Independent sample t-test found no statistically significant differences for any delirium measure across study sites

SD: Standard Deviation 


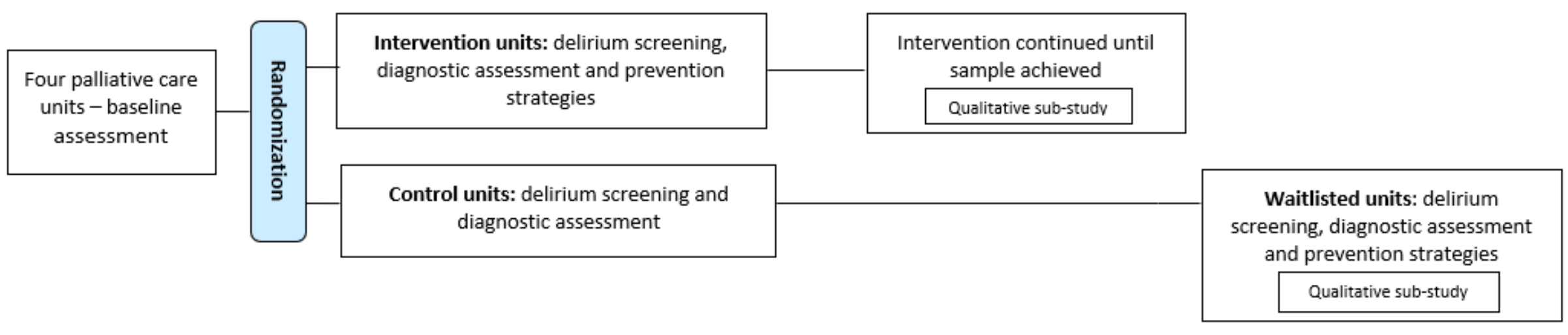

Figure 1: Study Diagram

Standardized delirium screening and diagnostic assessment at all units + delirium prevention strategies at

intervention units (May-Jun 2018); control units were waitlisted to the intervention (Aug-Sep 2018) 


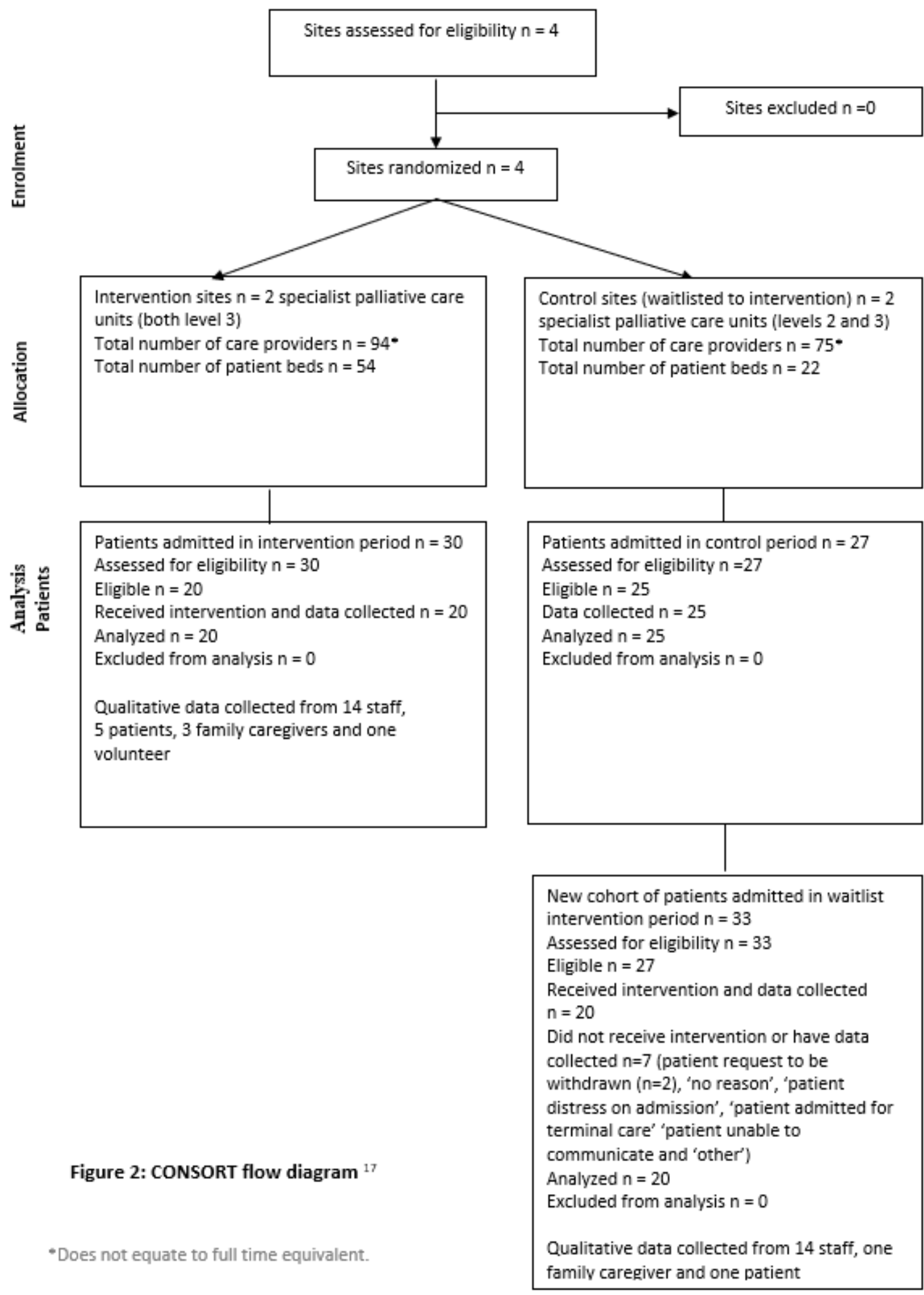




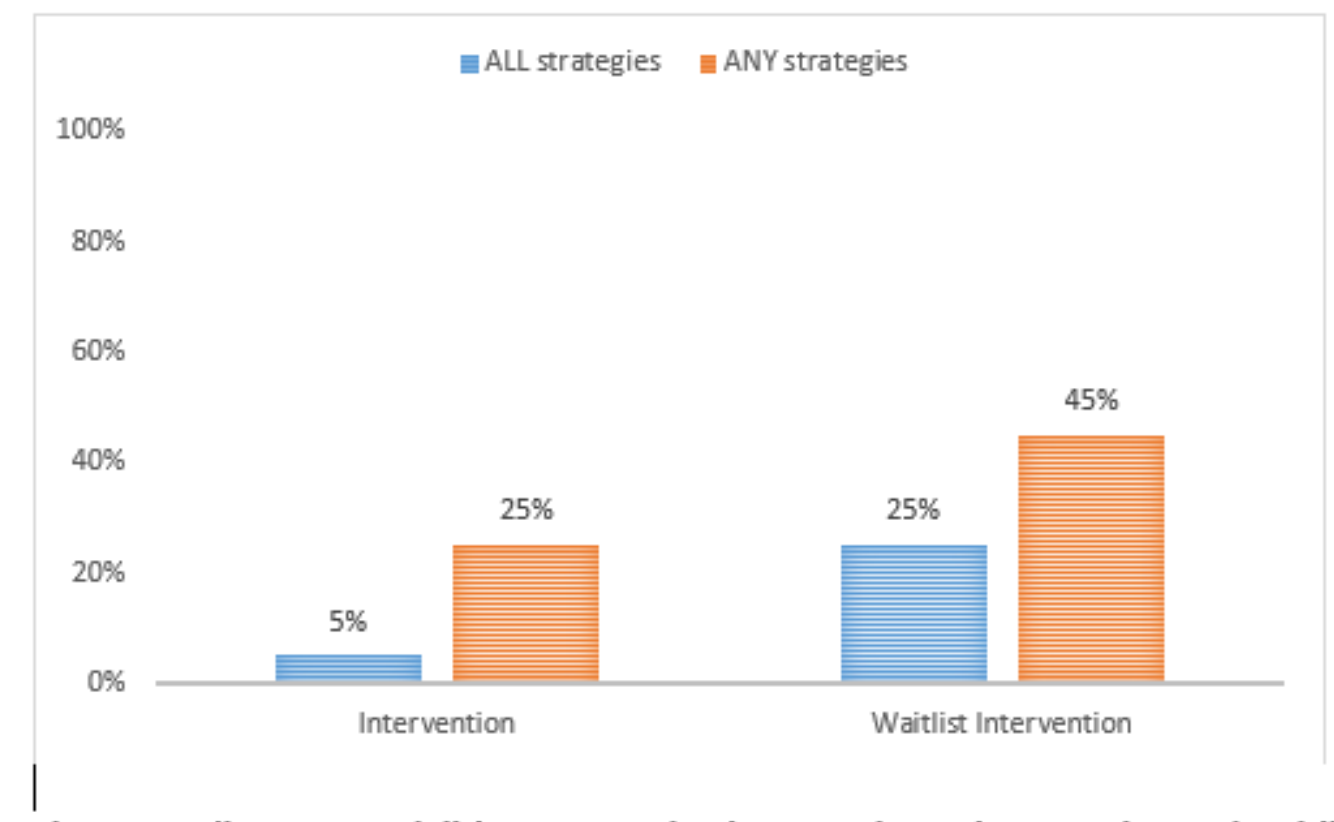

Figure 3: Adherence to delirium prevention intervention at intervention and waitlisted sites, calculated as either all strategies or any strategy $\geq 4$ domains for $\geq 5$ days. 


\section{Supplementary file 1: Elements of the intervention}

\begin{tabular}{|c|c|c|}
\hline Domains & Strategies & Implementation \\
\hline 1. Preserve natural sleep & $\begin{array}{ll}\text { - } & \text { Offer ear plugs to patients who have low risk of falls } \\
\text { - } & \text { Offer eye shades to patients who have low risk of falls } \\
\text { - } & \text { Reduce noise outside patient rooms during 21:00-06:00 } \\
\text { - } & \text { Normal day-night variation in room and unit lighting } \\
\text { - } & \text { Exposure to natural light during daylight hours } \\
\text { - } & \text { Schedule care activities to allow uninterrupted sleep during the night } \\
\text { - } & \text { Avoid caffeine after } 4 \mathrm{pm}\end{array}$ & 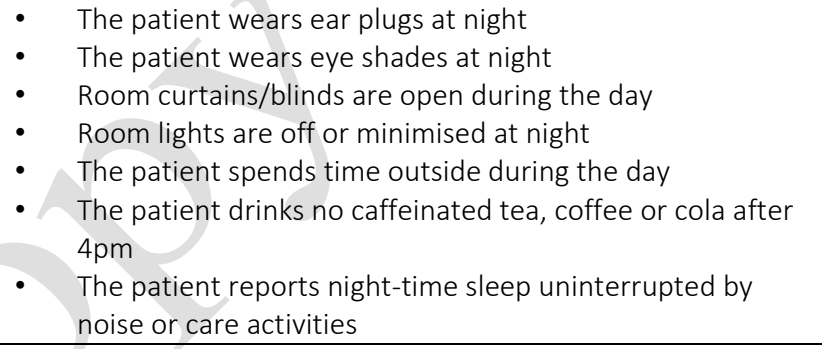 \\
\hline $\begin{array}{l}\text { 2. Maintain optimal sensory } \\
\text { perception }\end{array}$ & $\begin{aligned} & \text { Hearing: } \\
& \circ \text { Assess hearing } \\
& \circ \text { Assist with and re-inforce use of hearing aids and special } \\
& \text { communication techniques } \\
& \circ \text { Ear wax clearing as needed } \\
& \circ \quad \text { Vision: } \\
& \circ \text { Assess need for visual aids (glasses, magnifying lenses) } \\
& \circ \text { If needed, ask family to provide for the patient } \\
& \circ \text { Assist with and re-inforce use of visual aids } \\
&\end{aligned}$ & $\begin{array}{l}\text { - } \quad \text { The patient hearing was assessed } \\
\text { - The patient has ear wax cleaning } \\
\text { - The patient wears functioning hearing aids } \\
\text { - The patient has their vision assessed } \\
\text { - The patient uses visual aids }\end{array}$ \\
\hline 3. Optimize hydration & $\begin{array}{l}\text { - } \quad \text { Encourage oral fluids } \\
\text { - } \quad \text { Physical assistance with drinks and meals, as required } \\
\text { - } \quad \text { Drinking aids, as required } \\
\text { - } \quad \text { Be alert and respond to reversible causes of poor oral intake within } 24 \text { hours } \\
\text { e.g. nausea, vomiting, drowsiness, sore mouth. }\end{array}$ & $\begin{array}{l}\text { - The patient is encouraged to drink } \\
\text { - The patient is assisted with meals } \\
\text { - Drinking aids are provided e.g. straws, cup holders, cups } \\
\text { with lids, spouts, handles or grips } \\
\text { Intervention for reversible causes of poor oral intake are in } \\
\text { place }\end{array}$ \\
\hline $\begin{array}{l}\text { 4. Stimulate communication, } \\
\text { orientation and cognition }\end{array}$ & $\begin{array}{l}\text { - Communication: } \\
\text { Interpreter and translation for people with non-English speaking } \\
\text { background } \\
\text { Orientation: } \\
\circ \text { Greet the patient by name } \\
0 \text { Introduce self by name and role } \\
0 \text { Refer to person, time and place when talking with the patient } \\
0 \quad \text { Time aids in room e.g. watch, personal or wall clock; wall, desk or } \\
\text { electronic calendar } \\
\text { Update in-room whiteboards daily with date, day, place, reason for } \\
\text { admission, team member names, schedule } \\
0 \text { Minimize number of transfers to other beds or rooms within the unit } \\
\text { Cognition: } \\
0 \text { Discuss current events with the patient }\end{array}$ & $\begin{array}{l}\text { - Interpreter is available and utilized } \\
\text { - Orientating information is translated into the patient's } \\
\text { native language } \\
\text { - The patient can see the time in their room } \\
\text { - The patient can see the day, date and month in their room } \\
\text { unit } \\
\text { - The patient discusses current events } \\
\text { - The patient reminisces and/or talks about their life and } \\
\text { - Tamily } \\
\text { - } \text {-g. reading, puzzles, games, knitting, music } \\
\text { Cognitive stimulating activities are in the patient's care } \\
\text { plan }\end{array}$ \\
\hline
\end{tabular}




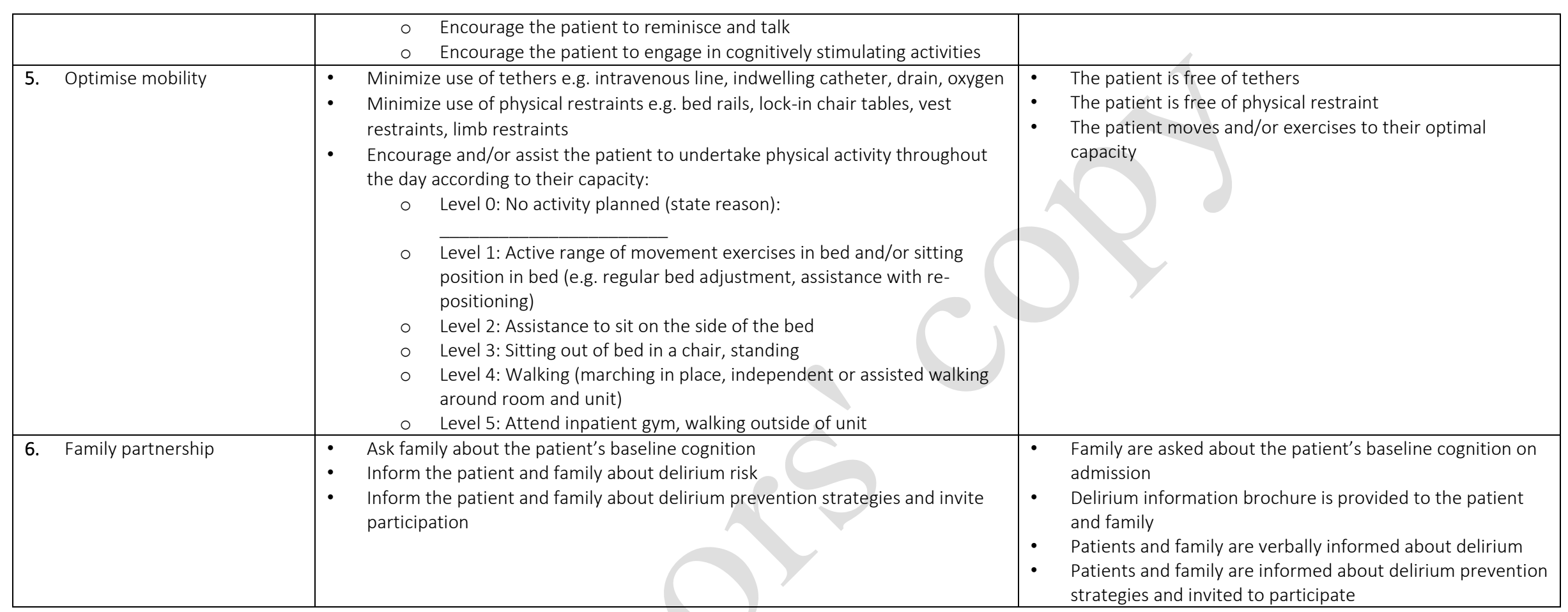


Supplementary file 2: Schedule of study measures and time points

\begin{tabular}{|c|c|c|c|c|c|c|c|c|}
\hline \multirow[t]{4}{*}{ Measure } & \multicolumn{8}{|c|}{ Study arm/period } \\
\hline & \multicolumn{5}{|c|}{ Control and intervention } & \multicolumn{3}{|c|}{ Intervention only } \\
\hline & \multirow[t]{2}{*}{ Baseline } & \multirow[t]{2}{*}{ O/A } & \multirow{2}{*}{$\begin{array}{l}\text { Daily } \\
(1-7)\end{array}$} & \multirow{2}{*}{$\begin{array}{l}\text { Nu-DESC } \\
\geq 2\end{array}$} & \multirow{2}{*}{$\begin{array}{l}\text { Study } \\
\text { end }\end{array}$} & \multirow{2}{*}{$\begin{array}{l}\text { Daily } \\
(1-7)\end{array}$} & \multicolumn{2}{|c|}{ Intervention completion } \\
\hline & & & & & & & $\begin{array}{l}\text { Immediately } \\
\text { after }\end{array}$ & $\begin{array}{l}\text { Six months } \\
\text { after }\end{array}$ \\
\hline \multicolumn{9}{|l|}{ Site level } \\
\hline Geographical location & $x$ & & & & & & & \\
\hline $\begin{array}{l}\text { Type and level of } \\
\text { service provision }\end{array}$ & $x$ & & & & & & & \\
\hline Number of beds & $x$ & & & & & & & 2 \\
\hline Team composition & $x$ & & & & & & & \\
\hline $\begin{array}{l}\text { Clinical documentation } \\
\text { method }\end{array}$ & $x$ & & & & & & & 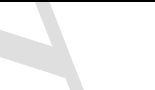 \\
\hline $\begin{array}{l}\text { Routine delirium } \\
\text { process and measures }\end{array}$ & $x$ & & & & & & & $x$ \\
\hline $\begin{array}{l}\text { Patient demographics, } \\
\text { function (AKPS, RUG- } \\
\text { ADL), palliative care } \\
\text { phase* }\end{array}$ & & & & & $x$ & & & \\
\hline \multicolumn{9}{|l|}{ Patient level } \\
\hline Primary diagnosis* & & $x$ & & & & & & $x$ \\
\hline Nu-DESC & & & $x$ & 8 & & & & $x$ \\
\hline $\begin{array}{l}\text { DSM-5 diagnostic } \\
\text { criteria for delirium }\end{array}$ & & & & $x$ & & & & $x$ \\
\hline DRS-R-98 & & & $\Delta$ & $x$ & & & & $x$ \\
\hline $\begin{array}{l}\text { Adherence to delirium } \\
\text { prevention strategies }\end{array}$ & & & & $\nabla$ & & $x$ & & $x$ \\
\hline \multicolumn{9}{|l|}{ Sub-study } \\
\hline $\begin{array}{l}\text { Semi-structured } \\
\text { interviews with } \\
\text { patients, family, staff } \\
\text { and volunteers }\end{array}$ & & & 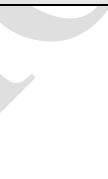 & & & & $x$ & \\
\hline
\end{tabular}

*Collected via Palliative Care Outcomes Collaborative (PCOC) routine measures for ALL admitted patients for study periods

AKPS: Australian-modified Karnofsky Performance Status; DRS-R-98: Delirium Rating Scale-Revised-1998; DSM-5:

Diagnostic and Statistical Manual of Mental Disorders, Fifth edition; Nu-DESC: Nursing Delirium Screening Scale; O/A: On admission; RUG-ADL: Resource Utilisation Groups-Activities of Daily Living 
Supplementary file 3: Adverse events

\begin{tabular}{|c|c|c|c|}
\hline Patient/event & $\begin{array}{l}\text { Control sites } \\
(n=25)\end{array}$ & $\begin{array}{l}\text { Intervention sites } \\
(n=20)\end{array}$ & $\begin{array}{l}\text { Waitlisted sites } \\
(n=20)\end{array}$ \\
\hline Patient n (\%) & $15(60)$ & $14(70)$ & $16(80)$ \\
\hline \multicolumn{4}{|c|}{ Category of adverse event $\mathrm{n}(\%)$} \\
\hline Falls $^{*}$ & $2(8)$ & $1(5)$ & $1(5)$ \\
\hline Complaints $^{*}$ & 0 & 0 & $1(5)$ \\
\hline Death $^{*}$ & $6(24)$ & $7(35)$ & $7(35)$ \\
\hline Other $^{+}$ & $12(48) ; 48(4)$ & $11(55) ; 38(3.5)$ & $15(75) ; 51(3.4)$ \\
\hline Serious $^{\dagger+}$ & $11(44) ; 15(1.4)$ & $6(30) ; 33(5.5)$ & $6(30) ; 33(5.5)$ \\
\hline \multicolumn{4}{|c|}{ Relatedness to intervention $^{* *}$} \\
\hline Unlikely & 0 & 3 (1 patient) & 0 \\
\hline Unrelated & 30 (15 patients) & 85 (12 patients) & 54 (14 patients) \\
\hline
\end{tabular}

* Reported as number (percentage) of patients who experienced the adverse event

+ Reported as number (percentage) of patients who experienced an adverse event other than falls, complaints or death; and overall number of other adverse events (mean number per patient)

†† Reported as number (percent) of patients who experienced a serious adverse event; and overall number of serious adverse events (mean number per patient)

**Other categories were Possible/Probably/Definite/Not possible, for which there were none 